\title{
Simulating Vibrational Energy Flow in Proteins: Relaxation Rate and Mechanism for Heme Cooling in Cytochrome c
}

\author{
Lintao Bu and John E. Straub* \\ Department of Chemistry, Boston University, Boston, Massachusetts 02215
}

Received: April 30, 2003; In Final Form: July 24, 2003

\begin{abstract}
The rate and mechanism of the kinetic energy relaxation of directly excited heme in cytochrome c was investigated using classical molecular dynamics simulation. The kinetic energy relaxation was found to be a biphasic exponential decay process with relaxation time constants of $1.5 \mathrm{ps}$ for the fast process and $10.1 \mathrm{ps}$ for the slow process. Approximately $60 \%$ of the kinetic energy relaxes on the short time scale. Energy flow from the heme to the protein is found to occur "through bond" via (1) covalent linkages of the heme to the Cys14, Cys17, His18, and Met80 protein residues and (2) hydrogen bonds to Tyr48, Thr49, Asn52, and Thr78 protein residues and nearby water molecules and "through space" via nonbonded collisional energy transfer to nearby heme pocket residues-Arg38, Thr40, Gly41, and Phe46. In a previous simulation study by Sagnella and Straub, heme "cooling" in myoglobin was found to proceed via a spatially anisotropic "funneling" mechanism as a single-exponential process with a time constant of 5.9 ps. Topological variations in heme/ protein connectivity and variations in the accessibility of the heme to the solvent are used to explain the distinctly different pathways and time scales for heme "cooling" in the two proteins.
\end{abstract}

\section{Background}

Heme proteins undergo vibrational energy relaxation (VER) following ligand binding or dissociation. To understand the time scales and mechanisms of vibrational energy transfer, it is essential to understand the conformational changes and reorganization of protein structures that follow fundamental events such as the binding or release of substrate molecules or electron transfer. ${ }^{1-18}$

An important goal of the detailed analysis of the vibrational relaxation of heme proteins is to provide important information about the cooperative nature of protein dynamics. ${ }^{7,8}$ In myoglobin, ligand dissociation can occur when the ligand-heme complex absorbs a visible or UV photon. Ligand photolysis is followed by vibrational excitation of the ligand, heme, and surrounding residues ${ }^{19,20}$ and a global conformational transformation of the protein itself. Experimental and theoretical studies have explored the rate of vibrational energy relaxation for small diatomic ligands, ${ }^{21-25}$ particularly $\mathrm{CO}$ in the heme protein myoglobin.

Computer simulation of vibrational energy relaxation in heme proteins began with the pioneering work of Henry, Eaton, and Hochstrasser, ${ }^{4}$ who found heme "cooling" in both myoglobin and cytochrome $\mathrm{c}$ to be a biphasic process with roughly $50 \%$ kinetic energy loss occurring in 1-4 ps and the remainder of the relaxation to thermal equilibrium occurring in $20-40 \mathrm{ps}$. The time scales observed for the relaxation of the heme were found to be approximately the same for both myoglobin and cytochrome $\mathrm{c}$. That result is surprising because the local environments of the heme are quite different in the two proteins.

More recent simulation studies of fully solvated myoglobin have provided a more detailed description of energy flow following ligand photolysis or the direct excitation of the heme. ${ }^{26,27}$ Simulations of the wild-type protein suggest that heme

* Corresponding author. E-mail: straub@bu.edu. vibrational relaxation is a single-exponential process with time constant of $5.9 \mathrm{ps}$, differing from the earlier simulation studies that suggested a biexponential relaxation process. ${ }^{4}$ Moreover, the recent studies suggested a mechanism of spatially anisotropic directed energy flow where the heme's isopropanoate side chains serve to "funnel" energy from the heme to the closely coupled solvent.

A recent simulation study by $\mathrm{Bu}$ and Straub of heme cooling in the H93G mutant, where the sole covalent bond between the heme $\mathrm{Fe}$ and protein is severed, showed no change in the relaxation rate or pathway of energy flow. ${ }^{27}$ That result is supported by an independent experimental study by Champion and co-workers, who found no difference in the rate of heme cooling in wild-type and $\mathrm{H} 93 \mathrm{G}$ mutant myoglobin. ${ }^{28}$ An additional simulation study of a modified heme, where the isopropanoate side chains were replaced by hydrogen atoms, showed a dramatic increase in the time scale for heme cooling by $50 \%$, providing support for the conjecture of Sagnella and Straub that the heme side chains play a particularly important role in the relaxation mechanism. ${ }^{27}$ Recent experimental results of Champion and co-workers indicated that the rate of heme cooling in myoglobin with porphine, a heme without side chains, was significantly slower relative to that of myoglobin with standard protoporphyrin IX, with side chains. ${ }^{28}$ These results lend support for the conjecture that heme cooling in myoglobin is a single-exponential process that occurs by spatially directed energy funneling from heme to solvent.

How does the rate and mechanism of heme cooling in cytochrome c differ from that in myoglobin? Differences in protein structure suggest that the time scale and mechanism of energy flow could be quite different in the two proteins. The heme chromophore in myoglobin has only one covalent bond to the protein - the bond between the Fe atom and a nitrogen atom of the proximal histidine, His93, shown in Figure 1. In the ligated state, the $\mathrm{Fe}$ is six coordinate, and the $\mathrm{Fe}-\mathrm{N}$ bond is orthogonal to the heme plane and its in-plane vibrational 

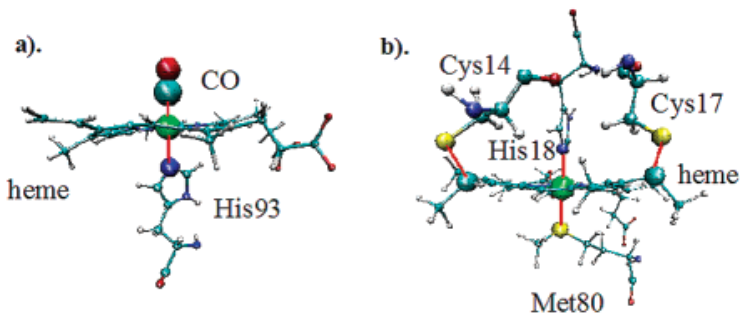

Figure 1. Active sites of (a) sperm whale myoglobin and (b) horse heart cytochrome c. In myoglobin, the heme is covalently bonded to the proximal His93 and the CO ligand. In cytochrome c, the heme group and residues His18 and Met80 ligate the heme's Fe atom. The heme is covalently attached to the apoprotein by two thioether linkages, formed by the addition of the thiol groups of two cysteine residues, Cys14 and Cys17, to the vinyl groups of the heme.

modes. This orientation decouples the $\mathrm{Fe}-\mathrm{N}$ stretch from the heme's in-plane vibrations, making through-bond energy transfer from in-plane heme vibrations through the proximal histidine to the surrounding protein an unlikely doorway for excess vibrational energy relaxation.

The environment of the heme chromophore in cytochrome $\mathrm{c}$ (cyt c) is quite different from that in myoglobin. Cytochrome $\mathrm{c}$ consists of a single polypeptide chain containing 104 amino acid residues and is organized into a series of $5 \alpha$ helices and $\operatorname{six} \beta$ turns. The heme active site in cytochrome $\mathrm{c}$ consists of a six-coordinate low-spin iron, binding His18 and Met80 as the axial ligands. In addition, two cysteines (Cys14 and Cys17) provide the thioether bridges to the heme shown in Figure 1. Crystal structures of cytochrome c show that the heme group is located in a groove and is almost completely buried inside the protein. The porphyrin is distinctly nonplanar and somewhat distorted into a saddle-shaped geometry. Therefore, throughbond transfer of excess kinetic energy from the heme to the protein following the direct excitation of in-plane heme vibrations is more likely in cytochrome $\mathrm{c}$ than in myoglobin.

We have used classical molecular dynamics simulation to observe the process of heme cooling in aqueously solvated cytochrome $\mathrm{c}$ following the direct excitation of the porphyrin chromophore. Although the results of previous studies indicate a single-exponential decay of heme excitation in myoglobin and several myoglobin variants, we observe that heme cooling in cytochrome $\mathrm{c}$ is a biphasic exponential function of time. We argue that the mechanism of heme cooling is different in cytochrome $\mathrm{c}$ and myoglobin and that the differences result from unique aspects of protein/heme topology in the two proteins.

\section{Computational Model and Methods}

2.1. Molecular Dynamics. An X-ray structure of the horse heart cytochrome c molecule ${ }^{29}$ was used as the initial configuration. That structure was introduced into a $55.872 \times 55.872$ $\times 55.872 \AA^{3}$ truncated octahedral box of equilibrated TIP3 water molecules and simulated using the CHARMM program ${ }^{30}$ following standard simulation protocol. ${ }^{26}$ The all-hydrogen parameter set (version 27) of the CHARMM force field ${ }^{31}$ was used. Equilibrated water molecules lying within $2.5 \AA$ of the protein molecule were removed, and the original water molecules of the X-ray structure were preserved. The excess potential energy due to bad contacts and strain was then reduced using the steepest-descent energy-minimization method.

Using classical molecular dynamics, the system was gradually heated to $300 \mathrm{~K}$. One molecular dynamics trajectory was run for $20 \mathrm{ps}$ at constant pressure and temperature, after which the volume of the box was found to fluctuate about a constant mean value. The new parameters for the box were found to be 53.934 $\times 53.934 \times 53.934 \AA^{3}$. Molecular dynamics trajectories were run for $200 \mathrm{ps}$ at constant energy and volume. During equilibration, the velocities were occasionally resampled according to the Maxwell statistical distribution of atomic velocities to maintain a constant temperature. Assuming that an equilibrium state had been reached, data were collected from the constant energy and volume dynamics.

The molecular dynamics trajectories were generated using the position Verlet algorithm, which is time-reversible and symplectic, ${ }^{32-34}$ employing a time step of $1 \mathrm{fs}$. The nonbonded potential was truncated using a group switching function, from 8.0 to $12.0 \AA$, applied to the Lennard-Jones interaction potential energy and a group-based switching function, from 8.0 to 12.0 $\AA$, which was applied to the electrostatic force. Snapshot configurations were saved every 20 ps during the 200-ps equilibration dynamics. From each of the 10 configurations obtained in this way, 30-ps trajectories were run for both equilibrium dynamics, continuing the NVE dynamics used for equilibration, and nonequilibrium dynamics, where the initial configuration for each excited state was identical to the corresponding nonexcited state at the moment of excitation, except for the velocities of the 75 heme atoms.

2.2. Simulation of Photon Absorption by the Heme. To simulate the absorption of a photon, approximately $68 \mathrm{kcal} /$ mol of excess kinetic energy, equivalent to one 420-nm photon (the largest absorption position in UV/vis spectroscopy of cytochrome c), was deposited in the heme. The excess kinetic energy on each atom was assigned as follows. The velocity of each atom of the porphyrin was randomly selected from a Maxwell statistical distribution. The magnitudes of the velocity components were then scaled to correspond to a net increase in energy equivalent to that due to the absorption of the photon. In prior studies of heme cooling in myoglobin, it was found that the intramolecular vibrational energy relaxation within the heme was sufficiently rapid that the results were insensitive to the exact means of distributing the excess energy within the heme. ${ }^{26}$

2.3. Measure of the Rate of Local Kinetic Energy Relaxation. The ergodic measure is a useful means of determining the time scale for the self-averaging of a given property in a many-body system. ${ }^{35-38}$ A particular form of the ergodic measure, the fluctuation metric, is defined to be

$$
\Omega(t)=\sum_{j}^{N}\left[f_{j}(t)-\bar{f}(t)\right]^{2}
$$

where $f_{j}(t)$ is the time average for atom $j$ of property $F(t)$ of the system and $\bar{f}(t)$ is the average of property $F(t)$ over all $N$ atoms at time $t$. Here we consider $f_{j}(t)$ to be the kinetic energy of the $j$ th atom. If the total system is self-averaging, then the function $\Omega_{\mathrm{KE}}(t)$ will decay to zero as $\Omega_{\mathrm{KE}}(t) / \Omega_{\mathrm{KE}}(0) \approx 1 / D_{\mathrm{KE}} t \rightarrow 0$. The slope of $\Omega_{\mathrm{KE}}(t)$ is proportional to the generalized diffusion constant $D_{\mathrm{KE}}$ for the kinetic energy. The mean square of the fluctuations in the kinetic energy metric is related to the generalized diffusion constant describing the rate of exploration within the kinetic energy state space. ${ }^{35,37}$

If we assume that the microscopic motion is well described by a Langevin dynamics model, then one can relate the rate of self-averaging of the kinetic energy to the magnitude of the static friction. ${ }^{38}$ The Langevin model is a "weak collision" model because it is based on the assumption that the exchange of energy with the bath takes place in increments that are small compared with the thermal energy. ${ }^{39,40}$ By evaluating the kinetic energy metric using dynamics consistent with the Langevin 


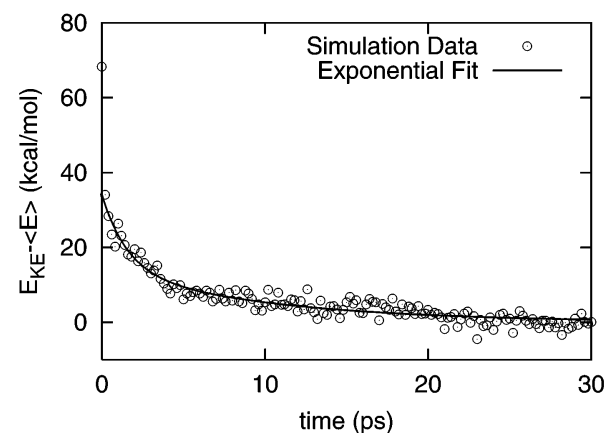

Figure 2. Exponential decay of the excess kinetic energy placed within the heme. The simulation data are represented by points, whereas the best exponential fit is represented by a solid line. The time decay of the kinetic energy can be modeled well by a biphasic exponential function with relaxation time constants of $1.5 \mathrm{ps}$ ( 0.58 weighting factor) and $10.1 \mathrm{ps}(0.42$ weighting factor), respectively. $\langle E\rangle$ is the average equilibrium kinetic energy for the heme at $300 \mathrm{~K}$, computed as $3 N k_{\mathrm{B}} T / 2$ where $N$ is the number of atoms in the heme.

TABLE 1: Thermal Energy Relaxation of the Heme Exhibited a Biphasic Decay for Both the Native Cytochrome c and Two Covalently Modified Cytochrome c Proteins $^{a}$

\begin{tabular}{lcc}
\hline \multirow{2}{*}{ molecule } & \multicolumn{2}{c}{ time constants $(\mathrm{ps})$} \\
\cline { 2 - 3 } \multicolumn{1}{c}{ fast process } & slow process \\
\hline native cytochrome $c$ & $1.5 \pm 0.3(58 \%)$ & $10.1 \pm 1.1(42 \%)$ \\
severed thioether bonds & $1.9 \pm 0.4(64 \%)$ & $12.0 \pm 1.7(36 \%)$ \\
severed Fe-S (Met80) bond & $1.7 \pm 0.3(55 \%)$ & $10.7 \pm 1.0(45 \%)$
\end{tabular}

${ }^{a}$ Respecting the simulation error, there is no significant difference between these protein molecules for the time scales of heme cooling and kinetic energy relaxation.

equation, we can estimate the generalized kinetic energy diffusion constant to be $D_{\mathrm{KE}}=\gamma_{0}$, where $\gamma_{0}$ is the static friction. It follows that at long times the slope of the ratio $\Omega_{\mathrm{KE}}(0) /$ $\Omega_{\mathrm{KE}}(t)$ is equal to the static friction constant $\gamma_{0}$. The computation of the kinetic energy metric is a straightforward and effective means of assigning time scales for atomic kinetic energy relaxation in molecular systems. ${ }^{38}$

\section{Results and Analysis}

The simulated dynamical trajectories were used to determine the time scales and pathways of the kinetic energy relaxation or cooling of the heme in cytochrome $\mathrm{c}$ following the absorption of a $420-n m$ photon.

3.1. Relaxation in Excited Cytochrome c. The average kinetic energy deposited over the 75 atoms in the heme during the excitation process is approximately $68 \mathrm{kcal} / \mathrm{mol}$. In our model, the simulated absorption induces an instantaneous increase in the temperature of the heme of over $300 \mathrm{~K}$. The average time dependence of the excess kinetic energy decay in the heme is shown in Figure 2. The simulation data are well fitted by a biphasic exponential decay function with relaxation time constants of $1.5 \mathrm{ps}$ for the fast process and $10.1 \mathrm{ps}$ for the slow process (Table 1). Approximately $60 \%$ of the excess energy was found to relax on the short time scale. The initial decay of the excess kinetic energy is rapid. The fast component is due to the energy transfer via the coupling between the heme and the collective motions of protein. The covalent bonds and hydrogen bonds between the heme and protein residues play the dominate role. The slow process is due to energy transfer via collisional contacts consistent with classical heat diffusion from the heme to the protein and to the solvent. ${ }^{41}$ In our simulation model, the excess kinetic energy is not deposited in a specific vibrational mode of the heme-it is redistributed

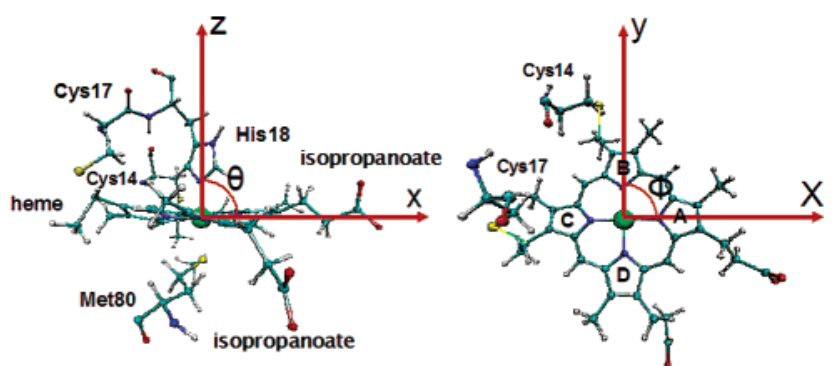

Figure 3. Heme shown with its $\mathrm{Fe}$ atom at the origin and nitrogen atoms of the pyrrole heme rings $\mathrm{A}$ and $\mathrm{B}$ located along the $x$ and $y$ axes, respectively. The Fe atom and the four nitrogen atoms are roughly in the $x-y$ plane. $\theta$ is the angle between the $z$ axis and the $x-y$ plane; $\phi$ is the angle measuring the rotation of the heme plane about the $z$ axis counterclockwise from the $x$ axis.

evenly among the heme atoms within a few femtoseconds. ${ }^{4,26}$ The rapid redistribution of the excess kinetic energy in the heme is consistent with the large value of the effective friction coefficients assigned to the atoms of the heme. ${ }^{38}$ Note that in the harmonic approximation the deposited excess kinetic energy will be redistributed equally between kinetic and potential energy. Therefore, the initial value of the kinetic energy decays to one-half of its original value, about $34 \mathrm{kcal} / \mathrm{mol}$.

3.2. Pathways for Kinetic Energy Relaxation. The spatial dependence of the pathway of kinetic energy relaxation was visualized in two ways. First, the angular distribution of the instantaneous kinetic energy of the atoms was projected in spherical polar coordinates. Second, instantaneous snapshots during the process of nonequilibrium relaxation were depicted using temperature-based color labels on all atoms. Our graphical analysis makes possible the identification of common features of relaxation pathways observed in the molecular dynamics trajectories.

3.2.1. Results for Wild-Type Cytochrome c. Figure 3 illustrates the orientation of the heme porphyrin with respect to angles $\theta$ and $\phi$. The coordinates of the system were translated and rotated so that the $\mathrm{Fe}$ atom sits at the origin and two nitrogen atoms of the heme's pyrrole rings $\mathrm{A}$ and $\mathrm{B}$ are positioned along the $x$ and $y$ axes, respectively. This transformation results in the Fe atom and the four pyrrole nitrogen atoms being positioned close to or in the $x-y$ plane. $\theta$ is the angle between the $z$ axis and the $x-y$ plane; $\phi$ is the angle measuring the rotation of the heme plane about the $z$ axis. Using this definition, the four pyrrolering nitrogen atoms were located near $\theta=90^{\circ}$ and $\phi$ values of $0,90,180$, and $270^{\circ}$ for rings $\mathrm{A}, \mathrm{B}, \mathrm{C}$, and $\mathrm{D}$, respectively.

In Figure 4, there are snapshots at six different times showing the positions (in $\theta$ and $\phi$ ) of atoms with excess kinetic energy that is significantly higher than the equilibrium average value. Both protein atoms and solvent atoms are shown. There is a high concentration of kinetic energy-a "hot spot" in the solvent-in the region of $\theta=90^{\circ}, \phi=180^{\circ}$. That is the location of the side chain of the heme ( $\mathrm{C}$ ring) where $\mathrm{Cys} 17$ is covalently coupled to the heme. There is a similarly distinct hot spot in the protein near $\theta=90^{\circ}, \phi=300^{\circ}$, which is the region of the interface between the protein and the $\mathrm{A}$ and $\mathrm{D}$ rings of the heme-the region of contacts between the protein and the heme's two isopropanoate side chains.

This plot clearly shows the spatially anisotropic-directed funneling of kinetic energy from the heme through its side chains into the surrounding solvent. These simulation results are consistent with the results of the earlier computational study of myoglobin, ${ }^{26,27}$ where spatially anisotropic energy flow during heme cooling was observed. Sagnella and Straub ${ }^{26}$ argued that the two side chains of the porphyrin (A and D rings) with 

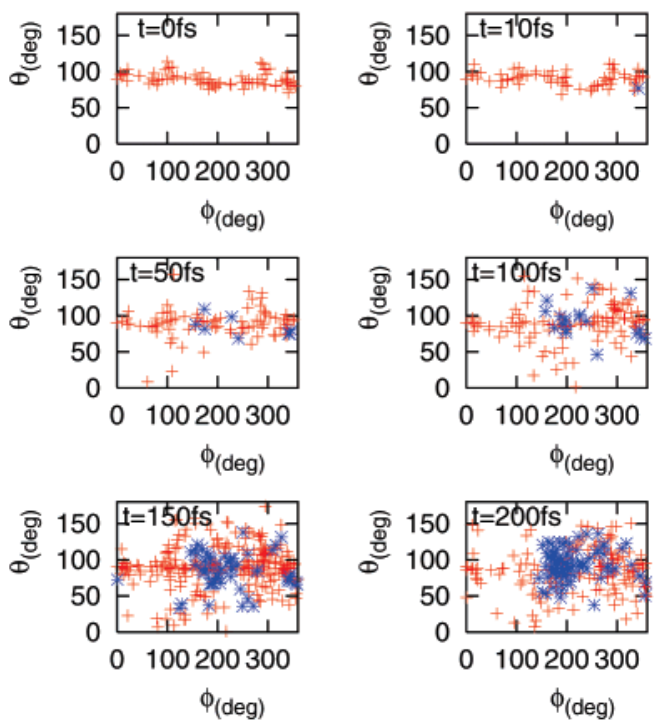

Figure 4. Redistribution of the excess kinetic energy in the hemecentered frame. All of the hot atoms with a significantly higher kinetic energy than the mean value at the equilibrium temperature are shown. Red + symbols represents the protein atoms, and blue $*$ symbols stand for the solvent atoms. The strong spatial anisotropy clearly demonstrates the directed funneling of excess kinetic energy through the heme side chains and into the surrounding solvent.

associated isopropanoate side chains form the dominant energytransfer pathway for kinetic energy relaxation. The results in Figure 4 indicate that whereas the energy flow is spatially anisotropic a larger fraction of the excess energy is transferred from the heme to the protein than was observed in myoglobin. This is due to the distinctly different protein topologies and connectivity to the heme. In particular, (1) the strong coupling of heme and protein in cytochrome $\mathrm{c}$ through direct covalent linkages and hydrogen bonds is responsible for the larger role of through-bond relaxation of the heme, and (2) the absence of the strong electrostatic interaction between the heme's isopropanoate side chains and the solvent is responsible for the smaller role of energy-funneling relaxation of the heme.

In Figure 5, we show snapshots of the vibrational energy redistribution in the heme and protein. Four sets of coordinates are shown. Heme atoms are shown using space-filling CPK models. The protein atoms and solvent atoms are indicated using dots. The atoms are colored by temperature. Red corresponds to the regions of highest temperature, and blue marks the regions of lowest temperature. The fluctuations in the kinetic energy are averaged over a time window of 50 fs to eliminate large fluctuations in the estimated instantaneous kinetic energy. That is to say, the "instantaneous" temperature shown at 50 fs corresponds to the average "temperature" in the first 50 fs for each atom; the temperature shown at $100 \mathrm{fs}$ corresponds to the temperature over the next $50 \mathrm{fs}$ for each atom and so on. It is evident that the heme is cooling over the time depicted. However, it is not possible to identify the pathway of energy transfer because the cooling process is very fast and the kinetic energy of the heme atoms drops precipitously. Because of the large number of degrees of freedom in the protein and solvent that are able to accept energy from the excited heme in our classical molecular dynamics simulations, the excess kinetic energy is redistributed over many protein atoms, and the average temperature of any given protein atom does not increase significantly.

As we show in Figure 6, the spreading of the kinetic energy from the heme to the protein occurs through two channels. One channel is through-space energy transfer by collisions of hot

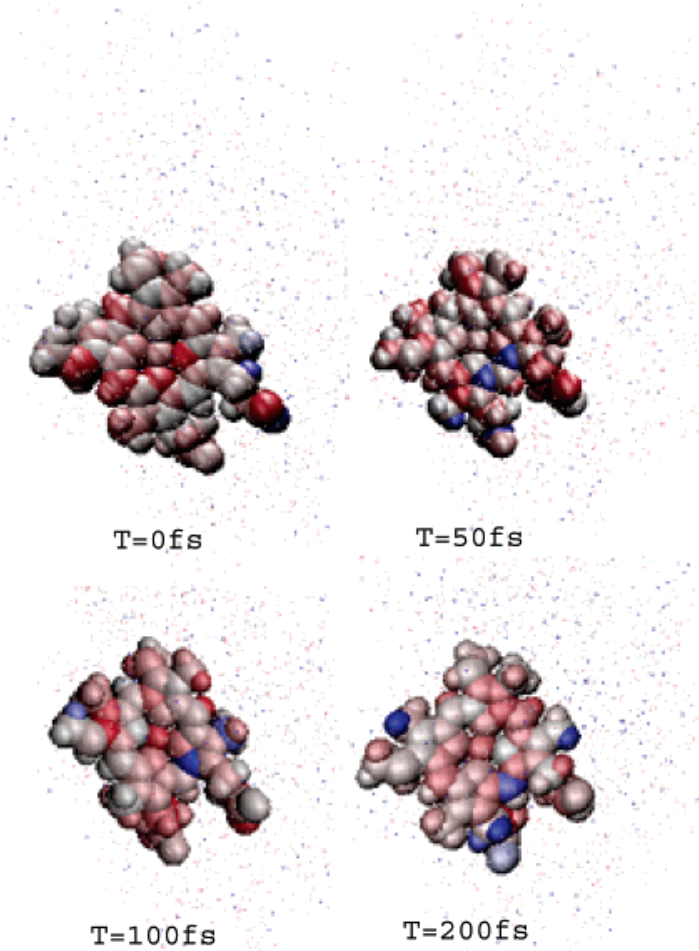

Figure 5. Redistribution of the excess kinetic energy from the heme to the surrounding protein. Only heme atoms are shown in space-filling CPK models. The protein atoms and solvent atoms are shown as dots. Each atom is colored on the basis of its temperature. Red corresponds to the regions of highest temperature, and blue marks the regions of lowest temperature.
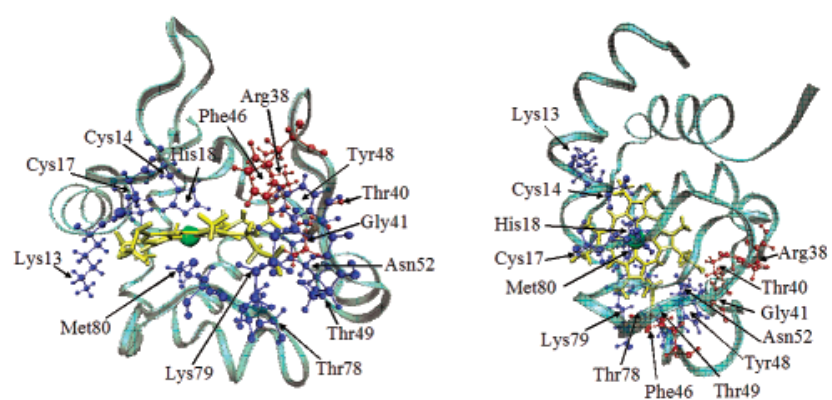

Figure 6. Hot protein residues in cytochrome $\mathrm{c}$ are depicted using a space-filling CPK model. Red indicates the through-space energytransfer channel including Arg38, Thr40, Gly41, and Phe46. Blue identifies the through-bond energy-transfer channel consisting of (1) Cys14, Cys17, His18, and Met80 (covalently bonded to heme), (2) Tyr48, Thr49, Asn52, and Thr78 (hydrogen- bonded to heme) and (3) Lys13 and Lys79 (neighboring residue of those in 1 or 2).

heme atoms with surrounding protein atoms. Another channel is through-bond energy transfer by intramolecular vibrational relaxation through the covalent bonds of the heme atoms to the protein atoms. After the first 50 fs following direct excitation, the protein residues contain hot atoms with an instantaneous kinetic energy that is significantly higher than average. The regions of the protein that accept significant quantities of kinetic energy can be divided into two sets-one contains Lys13, Cys14, Cys17, His18, Tyr48, Thr49, Asn52, Thr78, Lys79, and Met80, and the other consists of Arg38, Thr40, Gly41, and Phe46. The first set is composed of residues that are bonded to the heme. Cys14, Cys17, His18, and Met80 are covalently bonded to the heme, and Tyr48, Thr49, Asn52, and Thr78 are associated with the heme through hydrogen bonds, as shown in Figure 7 . The residues in the second set are those in close proximity, but not 


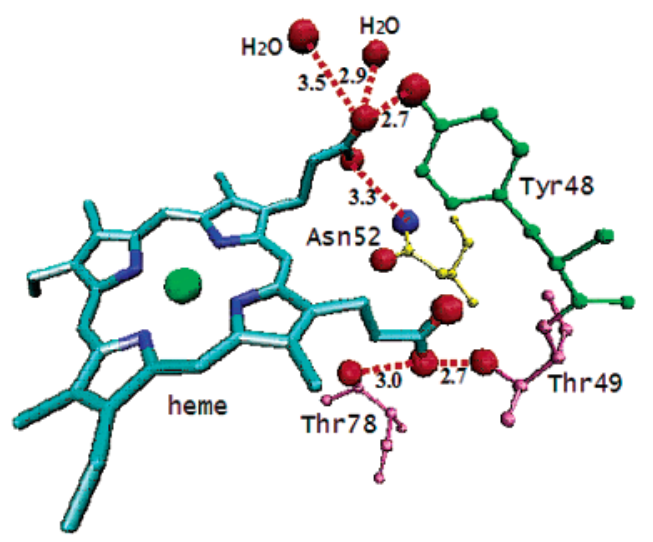

Figure 7. Protein residues hydrogen bonded to the heme are depicted using space-filling CPK models. Red indicates oxygen atoms, and nitrogen atoms are blue. The hydrogen bond length is determined from the initial crystal structure.

directly bonded, to the heme. The first set of residues forms the through-bond energy-transfer channel, and the second set forms the through-space energy-transfer channel. It should be noted that our definition is based on the behavior of the atoms at the onset of energy transfer from the heme. As time increases, it is practically impossible to make a clean separation between the two energy-transfer channels.

3.2.2. Modified Cytochrome c with Severed Bonds to Cys 14 and Cys17. Our simulation results suggest that the throughbond energy-transfer channel is more important in cytochrome $\mathrm{c}$ than in myoglobin, partially because there are four covalent bonds and four hydrogen bonds between the heme porphyrin and protein residues in cytochrome c, compared with a single covalent bond between the porphyrin and protein in myoglobin. Moreover, two of the covalent bonds between the protein and heme in cytochrome $\mathrm{c}$ are essentially in-plane to the heme and can be expected to be coupled to in-plane vibrational modes excited by ligand photolysis. To estimate the role of the covalent bonds in vibrational energy relaxation, we made a modified cytochrome c molecule in which we severed the two thioether bridges between the heme and the protein. In the modified protein molecule, there are only two covalent bonds-those between the heme Fe atom and protein residues His18 and Met80. (The four hydrogen bonds between the heme and protein are not changed.) Our protocol for the simulation of this modified cytochrome $\mathrm{c}$ molecule was identical to that described earlier for simulations of the native cytochrome $\mathrm{c}$.

Just as we observed in the case of the native cytochrome c protein, the kinetic energy relaxation in the modified cytochrome $\mathrm{c}$ is also a biphasic process. The relaxation time constants are $1.9 \mathrm{ps}$ for the fast process and $12.0 \mathrm{ps}$ for the slow process. These results are shown in Table 1 . The rate of kinetic energy transfer from the heme in the native cytochrome $\mathrm{c}$ is marginally faster than in the modified cytochrome c molecule due to the covalent bonds to the heme. Although our results point to a slowing of the rate of heme cooling, the relaxation times for the native and modified cytochrome $\mathrm{c}$ molecules are within the estimated uncertainty. Our results imply, but do not definitively demonstrate, the essential role of the covalent bonds between the protein and porphyrin in the relaxation process. We reason that the similarity in the heme cooling kinetics is due to the fact that there are four covalent bonds and six hydrogen bonds between the heme and its surroundings in the native proteinfour hydrogen bonds between the protein and heme and two hydrogen bonds between the heme and solvent. With such strong direct through-bond connectivity of the heme, our classical molecular dynamics simulation indicates that the cleavage of only two covalent bonds bonds between the heme and protein does not influence the cooling process significantly.

3.2.3. Modified Cytochrome $c$ with a Severed Bond to Met80. To explore the role of through-bond energy transfer further, we observed heme cooling in simulations of a modified cytochrome $\mathrm{c}$ where the covalent bond between the heme's iron atom and the sulfur atom of the Met80 residue was severed. The results of our simulations of the modified system suggest that the heme cooling in cytochrome $\mathrm{c}$ following Met80 ligand photolysis remains a biphasic process. The relaxation time constants were found to be $1.7 \mathrm{ps}$ for the fast process and $10.7 \mathrm{ps}$ for the slow process (Table 1). The time scales for both the fast and slow relaxation processes are slower than the time scales for the native cytochrome $\mathrm{c}$ and faster than those observed for the modified cytochrome $\mathrm{c}$ lacking thioether bonds between the two cysteine residues, Cys14 and Cys17, and the heme. The qualitative trends in these results are reasonable. Because the most effective modes active in the dissipation of kinetic energy from the heme are in-plane vibrations, those modes are weakly coupled to the outof-plane direction of the bond between Met80 and the heme. That result and interpretation is consistent with the result for myoglobin showing that heme cooling in the His93Gly mutant was identical to that observed for the native protein to within the uncertainty of the computed value. ${ }^{27}$

The time scale for the fast component of the vibrational relaxation of the heme is also consistent with the experimental results of Champion and co-workers, who observed vibrational cooling in cytochrome $\mathrm{c}$ following Met80 ligand photolysis in the range of $0.1-2.8 \mathrm{ps}^{42}$ In the experimental study, there is strong evidence for the photodissociation of the Met80 ligand from the heme following excitation. Rebinding of the Met80 ligand occurs on the time scale of $6.2 \mathrm{ps}$. The computational model employed in our studies does not allow for the photodissociation or rebinding of the Met80 ligand from the heme. However, because we find essentially no statistically significant difference between the time scales for heme cooling in the native and modified (dissociated Met80) proteins, we reason that our simulations are consistent with the results of the experimental study of Champion and co-workers. ${ }^{42}$

3.3. Spatial Anisotropy in Kinetic Energy Relaxation. The inverse kinetic energy fluctuation metric $\Omega_{\mathrm{KE}}(0) / \Omega_{\mathrm{KE}}(t)$ was computed as a function of time for the total system - the protein, the heme, and the solvent. The simulations were run at $300 \mathrm{~K}$. Each data set consists of an average over 10 30-ps nonequilibrium molecular dynamics trajectories. Values of the effective intrinsic friction constant, $\gamma_{0}$, were derived using the relation that at long time the ratio $\left[\Omega_{\mathrm{KE}}(0) / \Omega_{\mathrm{KE}}(t)\right]$ is equal to the static friction $\gamma_{0}$. In Figure 8, the function $\Omega_{\mathrm{KE}}(t)$ for the solvated cytochrome c system is plotted as a function of time. For all subsystems, $\Omega_{\mathrm{KE}}(t)$ decays to zero for each of the components listed, demonstrating the expected ergodic sampling of the momenta on the simulation time scale.

In Figure 9, the function $\Omega_{\mathrm{KE}}(0) / \Omega_{\mathrm{KE}}(t)$ for the solvated cytochrome c system is plotted as a function of time. Data for the individual contributions of the protein, the heme, and the solvent are listed in Table 2. These data illustrate the relative amount of damping felt by the atoms within the indicated subsets of the solvated cytochrome c system. The frictional forces felt by the heme are considerably greater than those acting on the remainder of the system. In fact, the effective friction constant for atoms of the heme is approximately 4 times larger than that for the protein itself. 


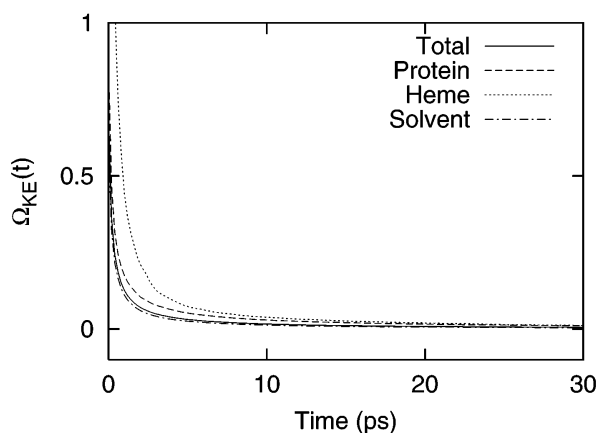

Figure 8. $\Omega_{\mathrm{KE}}(t)$ plotted as a function of time for the solvated cytochrome c system-the protein, the heme, and the solvent. For the convergence of ergodic sampling, $\Omega_{\mathrm{KE}}(t)$ decays to zero with time. The calculation represents an average over the 10 trajectories for the equilibrium NVE molecular dynamics of the aqueously solvated cytochrome c protein.

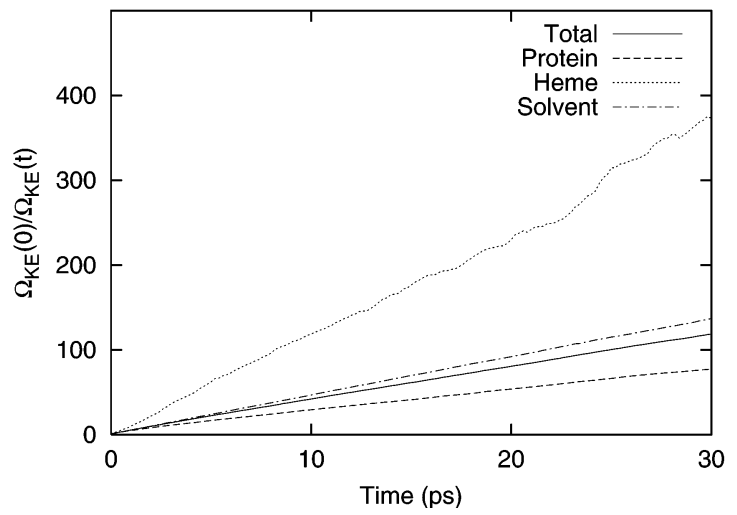

Figure 9. $\Omega_{\mathrm{KE}}(0) / \Omega_{\mathrm{KE}}(t)$ plotted as a function of time for the solvated cytochrome c system - the protein, the heme, and the solvent. The data were computed from an average over the 10 trajectories for the equilibrium NVE molecular dynamics of the aqueously solvated cytochrome c protein.

TABLE 2: Rate of Convergence of the Kinetic Energy Metric Interpreted as a Static Friction Constant, through a Langevin Dynamics Model, for Simulations of Aqueously Solvated Cytochrome c and Myoglobin ${ }^{a}$

\begin{tabular}{lcccc}
\hline & \multicolumn{2}{c}{ cytochrome $\mathrm{c}$} & & H93G myoglobin \\
\cline { 2 - 3 } region & $1 / \gamma_{0}(\mathrm{ps})$ & $\gamma_{0}\left(\mathrm{ps}^{-1}\right)$ & & $\gamma_{0}\left(\mathrm{ps}^{-1}\right)$ \\
\hline total & $0.25 \pm 0.01$ & $4.03 \pm 0.09$ & & $3.83 \pm 0.10$ \\
protein & $0.37 \pm 0.02$ & $2.69 \pm 0.16$ & & $2.62 \pm 0.18$ \\
heme & $\mathbf{0 . 0 8} \pm \mathbf{0 . 0 2}$ & $\mathbf{1 2 . 0} \pm \mathbf{2 . 8}$ & & $\mathbf{6 . 3 7} \pm \mathbf{1 . 0 5}$ \\
solvent & $0.22 \pm 0.01$ & $4.61 \pm 0.10$ & & $4.47 \pm 0.10$
\end{tabular}

${ }^{a}$ Here, total means the entire system consisting of the cytochrome c protein, the heme, and the solvent.

In His93Gly mutant myoglobin, the time constants calculated as the reciprocal intrinsic friction constant for the protein, the heme, and the solvent were found to be $0.38,0.16$, and 0.22 ps, respectively. ${ }^{27}$ The time scales for the heme and solvent are similar, whereas the time scale for the protein is approximately a factor of 2 longer in His93Gly mutant myoglobin. These results suggest that the solvent could play a significant role in the flow of energy out of the heme in myoglobin. However, the situation in cytochrome $\mathrm{c}$ is somewhat different from that in myoglobin. The average friction constant for atoms of the heme is much larger than for the remainder of the system. Although the effective friction acting on the solvent atoms is larger than that for the protein atoms, the difference is not great. This is due to differences in the local environment of the heme in these two proteins. In myoglobin, the two isopropanoate side chains extend out of the protein, forming a highly solvated region. Therefore, the kinetic energy can be transferred from the heme to the solvent rapidly. In cytochrome $\mathrm{c}$, the two isopropanoate side chains remain inside the protein, forming contacts with proximate polar residues including Tyr48, Thr49, Asn52, and Thr78 (Figure 7), and the two ethyl side chains extend out of the protein. Unlike the isopropanoate groups, the ethyl groups are not highly solvated in an aqueous environment. As a result, the channel of direct energy transfer through the side chains is much less effective, and therefore less important, in cytochrome $\mathrm{c}$ than in myoglobin.

\section{Summary and Conclusions}

Cytochrome $\mathrm{c}$ is one of the most thoroughly physicochemically characterized metalloproteins. ${ }^{43}$ We have used classical molecular dynamics simulations to investigate the vibrational energy relaxation of the heme porphyrin in room-temperature aqueously solvated cytochrome $\mathrm{c}$ following the direct excitation of the heme by the simulated absorption of a photon. Our simulations suggest that in the native wild-type protein the heme's kinetic energy relaxes according to a biphasic exponential decay process with relaxation time constants of $1.5 \mathrm{ps}$ for the fast process and $10.1 \mathrm{ps}$ for the slow process. The simulation also indicates that there is a spatially directed energy flow through the side chains of the heme to the surrounding solvent.

The dominant channel for the relaxation of the excess kinetic energy of the heme consists of two steps. First, the excess kinetic energy is rapidly redistributed within the heme atoms. Subsequently, the excess kinetic energy is transferred from the heme to the protein and the neighboring solvent molecules. (1) The through-bond energy transfer initially occurs from the heme to the proximal His 18 and Met80 that are covalently bonded to the heme iron atom, to the Cys14 and Cys17 residues that are bonded to the heme side chains, and to protein residues Tyr48, Thr49, Asn52, and Thr78 through hydrogen bonds. (2) The through-space energy transfer proceeds through nonbonded collisional energy transfer to nearby heme pocket residues such as Arg38, Thr40, Gly41, and Phe46. (3) The direct energy transfer to the solvent is minimized by the absence of the strong interaction between the heme's isopropanoate side chains and the solvent.

For cytochrome $\mathrm{c}$ in aqueous solution, our results indicate that through-bond channels play a dominate role in the heme cooling process. That result stands in contrast to the results of our previous simulation studies of heme cooling in myoglobin. ${ }^{26,27}$ In cytochrome $\mathrm{c}$, heme cooling appears to be a biexponential process with fast $(<2 \mathrm{ps})$ relaxation associated with rapid relaxation through bonds via modes coupled to the protein and slow ( $>10 \mathrm{ps}$ ) relaxation associated with collisional energy transfer that can be viewed as thermal diffusion from the heme to the protein and the solvent. In myoglobin, we have observed heme cooling to occur by a single-exponential decay process (with an intermediate time constant of $5.9 \mathrm{ps}$ ); ${ }^{26,27}$ there is substantial evidence for the spatially directed funneling of excess kinetic energy from the heme directly to the solvent, which acts as the dominant energy-transfer channel. In cytochrome c, energy flow is directed through two channels - a fast process through bonds and a slower process through space. Because of the weak interaction between the heme side chains and surrounding solvent molecules in cytochrome $\mathrm{c}$, the rate of the slow process decreased significantly relative to that of myoglobin (6 ps in myoglobin and $>10 \mathrm{ps}$ in cytochrome c).

We identify three principal causes for the differences in the rates of heme cooling observed in our classical molecular 
dynamics calculations for cytochrome c and myoglobin. (1) The different topological structures of the hemes lead to substantial through-bond coupling between the heme and protein in cytochrome $\mathrm{c}$ that is essentially absent in myoglobin. In myoglobin, the heme is predominately planar in the sixcoordinate carboxymyoglobin and only moderately domed in the five-coordinate deoxymyoglobin. There is no measurable energy flow from the heme through the covalent bond to the proximal histidine. In cytochrome $\mathrm{c}$, the heme is saddle-shaped and substantially nonplanar in the six-coordinate species as well as in the five-coordinate heme following the dissociation of Met80. Although there is no substantial energy flow through the covalent bond to Met80, there is measurable energy flow through the in-plane bonds to Cys14 and Cys17. (2) The different heme-protein connectivity (a single covalent bond between the heme and protein in myoglobin and four covalent bonds between the heme and protein in cytochrome c) provides a mechanism of fast energy transfer in cytochrome $\mathrm{c}(<2 \mathrm{ps})$ that is absent in myoglobin. (3) Direct coupling of the heme to the solvent through charged side chains in myoglobin, which leads to a highly directed energy-funneling mechanism of heme cooling in myoglobin, is largely absent in cytochrome c. This comparative analysis provides a consistent picture of heme cooling in these fundamentally important proteins.

Acknowledgment. We thank R. J. Dwayne Miller and Paul Champion for helpful comments. We are grateful for the generous support of this research by the National Science Foundation (CHE - 0316551).

\section{References and Notes} 162

(1) Muench, E.; Champion, P. Ann. N.Y. Acad. Sci. 1975, 24, 142-

(2) Greene, B.; Hochstrasser, R.; Weisman, R.; Eaton, W. Proc. Natl. Acad. Sci. U.S.A. 1978, 75, 5255-5259.

(3) Frauenfelder, H.; Wolynes, P. Science 1985, 229, 337-345.

(4) Henry, E.; Eaton, W.; Hochstrasser, R. Proc. Natl. Acad. Sci. U.S.A. 1986, 83, 8982-8986.

(5) Elber, R.; Karplus, M. Science 1987, 235, 318-321.

(6) Elber, R.; Karplus, M. J. Am. Chem. Soc. 1990, 112, 9161-9175.

(7) Miller R. J. D. Annu. Rev. Phys. Chem. 1991, 42, 581-614.

(8) Miller R. J. D. Acc. Chem. Res. 1994, 27, 145-150.

(9) Straub, J.; Karplus, M. Chem. Phys. 1991, 158, 221-248. 17916

(10) Li, H.; Elber, R.; Straub, J. J. Biol. Chem. 1993, 268, 17908-

(11) Onuchic, J.; Wolynes, P. J. Chem. Phys. 1993, 98, 2218-2224.

(12) Lim, M.; Jackson, T.; Anfinrud, P. Science 1995, 269, 962-966.

(13) Gnanakaran, S.; Hochstrasser, R. J. Chem. Phys. 1996, 105, 34863496.

(14) Zewail, A. J. Phys. Chem. 1996, 100, 12701-12712.
(15) Mizutani, Y.; Kitagawa, T. Science 1997, 278, 443-446.

(16) Park, E.; Thomas, M.; Boxer, S. J. Am. Chem. Soc. 2000, 122 12297-12303.

(17) Sage, J.; Paxson, C.; Wyllie, G.; Sturhahn, W.; Durbin, S.; Champion, P.; Alp, E.; Scheidt, W. J. Phys.: Condens. Matter 2001, 13 , $7707-7722$.

(18) Sage, J.; Durbin, S.; Sturhahn, W.; Wharton, D.; Champion, P.; Hession, P.; Sutter, J.; Alp, E. Phys. Rev. Lett. 2001, 86, 4966-4969.

(19) Kholodenko, Y.; Volk, M.; Gooding, E.; Hochstrasser, R. Chem. Phys. 2000, 259, 71-87.

(20) Asplund, M.; Zanni, M.; Hochstrasser, R. Proc. Natl. Acad. Sci. U.S.A. 2000, 97, 8219-8224.

(21) Anfinrud, P.; Han, C.; Hochstrasser, R. Proc. Natl. Acad. Sci. U.S.A. 1989, 86, 8387-8391.

(22) Hill, J.; Dlott, D.; Rella, C.; Peterson, K.; Decatur, S.; Boxer, S.; Fayer, M. J. Phys. Chem. 1996, 100, 12100-12107.

(23) Ma, J.; Huo, S.; Straub, J. J. Am. Chem. Soc. 1997, 119, 25412551.

(24) Meller, J.; Elber, R. Biophys. J. 1998, 74, 789-802.

(25) Okazaki, I.; Hara, Y.; Nagaoka, M. Chem. Phys. Lett. 2001, 337, $151-157$.

(26) Sagnella, D.; Straub, J. J. Phys. Chem. B 2001, 105, 7057-7063.

(27) Bu, L.; Straub, J. J. Phys. Chem. B 2003, 107, 10634-10639.

(28) Ye, X.; Demidov, A.; Rosca, F.; Wang, W.; Kumar, A.; Ionascu, D.; Zhu, L.; Barrick, D.; Wharton, D.; Champion, P. J. Phys. Chem. B, in press.

(29) Bushnell, G.; Louie, G.; Brayer, G. J. Mol. Biol. 1990, 214, 585595.

(30) Brooks, B.; Bruccoleri, R.; Olafson, B.; States, D.; Swaninathan, S.; Karplus, M. J. Comput. Phys. 1983, 4, 187-217.

(31) MacKerell, A. D., Jr.; Bashford, D.; Bellott, M.; Dunbrack, R. L., Jr.; Evanseck, J. D.; Field, M. J.; Fischer, S.; Gao, J.; Guo, H.; Ha, S.; Joseph-McCarthy, D.; Kuchnir, L.; Kuczera, K.; Lau, F. T. K.; Mattos, C.; Michnick, S.; Ngo, T.; Nguyen, D. T.; Prodhom, B.; Reiher, W. E., III; Roux, B.; Schlenkrich, M.; Smith, J. C.; Stote, R.; Straub, J.; Watanabe, M.; Wiórkiewicz-Kuczera, J.; Yin, D.; Karplus, M. J. Phys. Chem. B 1998, $102,3586-3616$.

(32) Verlet, L. Phys. Rev. 1967, 159, 98-103.

(33) Tuckerman, M.; Berne, B.; Martyna, G. J. Chem. Phys. 1992, 97, 1990-2001.

(34) Frenkel, D.; Smit, B. Understanding Molecular Simulation: From Algorithms to Applications, 2nd ed.; Academic Press: New York, 2001

(35) Thirumalai, D.; Mountain, R. Phys. Rev. A 1990, 42, 4574-4587.

(36) Straub, J.; Thirumalai, D. Proc. Natl. Acad. Sci. U.S.A. 1993, 90, 809-813.

(37) Straub, J.; Rashkin, A.; Thirumalai, D. J. Am. Chem. Soc. 1994, 116, 2049-2063.

(38) Sagnella, D.; Straub, J.; Thirumalai, D. J. Chem. Phys. 2000, 113, $7702-7711$

(39) Straub, J.; Borkovec, M.; Berne, B. J. Chem. Phys. 1988, 89, 48334847.

(40) Berne, B.; Borkovec, M.; Straub, J. J. Phys. Chem. 1988, 92, 37113725 .

(41) Lian, T.; Locke, B.; Kholodenko, Y.; Hochstrasser, R. J. Phys. Chem. 1994, 98, 11648-11656.

(42) Wang, W.; Ye, X.; Demidov, A.; Rosca, F.; Sjodin, T.; Cao, W. Sheeran, M.; Champion, P. J. Phys. Chem. B 2000, 104, 10789-10801. 521.

43) Sivakolundu, S.; Mabrouk, P. J. Am. Chem. Soc. 2000, 122, 1513- 